\title{
A SHEAF REPRESENTATION OF DISTRIBUTIVE PSEUDOCOMPLEMENTED LATTICES
}

\author{
WILLIAM H. CORNISH
}

\begin{abstract}
The main result of this paper shows that a distributive pseudocomplemented lattice $(L ; \vee, \wedge, *, 0,1)$, considered as an algebra of type $\langle 2,2,1,0,0\rangle$, can be represented as the algebra of all global sections in a certain sheaf. The stalks are the quotient algebras $L / \Theta(O(P))$, where $P$ is a prime ideal in $L$. The base space is the set of prime ideals of $L$ equipped with the topology whose basic open sets are of the form $\{P: P$ prime in $L, x * * P\}$ for some $x \in L$.
\end{abstract}

Introduction. In [2] the author considered the kernels of *-congruences on a distributive pseudocomplemented lattice $L$ and the properties of the smallest *-congruence. $\Theta(J)$, having a congruence-kernel, $J$, as its kernel. Though such congruences $\Theta(J)$ are only very well-behaved in the case where $L$ is a Stone lattice [2, Theorem 3.2], they, nevertheless, have enough properties to ensure that $L$ may be represented as a subdirect product, which is dependent on the variety $\mathscr{B}_{n}(1 \leqslant n<\omega)$ of a distributive pseudocomplemented lattice in which $L$ is found [2, Corollary 2.9]. The subdirect representation is not as simple as one might have wished. However, it owes its existence to the fact that the congruences $\Theta(J)$ can be associated with nice congruences connected with certain filters, cf. Theorem 1.5 and also the proof of (ii) in Lemma 2.4 of [2].

The main aim of this paper is to convert the aforementioned subdirect representation into a sheaf representation in which the lattice is represented as the algebra of all global sections. Our answer is not as pleasant as we might have wished. The base space of the sheaf turns out to be the set of all prime ideals of the lattice, endowed with an unfamiliar topology. However, the fact that the subdirect representation is converted to a sheaf-theoretic one in which the algebra becomes all global sections is not without interest. Indeed, B. A. Davey $[5, \S 2]$ has shown that any subdirect representation of an arbitrary universal algebra yields a sheaf-theoretic one but, of course, one cannot expect that the algebra will become the algebra of all global sections.

The kernels of *-congruences of a distributive pseudocomplemented lattice $L$ are precisely the $\alpha$-ideals of the distributive lattice, in the sense of [1]. Thus, a secondary aim of this paper is to examine the connection between $\alpha$-ideals and filters. We do this, together with other preliminary results, in $\S 1$ and give the sheaf representation in $\$ 2$.

Received by the editors January 27, 1975.

AMS (MOS) subject classifications (1970). Primary 06A35.

Key words and phrases. Distributive pseudocomplemented lattice, sheaf representation, $\alpha$-ideal, congruences. 
1. Congruences. Throughout this paper all lattices will be distributive with 0 and 1, and our notation and terminology follow that of [1], [2] and [3]. From [1, Proposition 3.3] it follows that an ideal $J$ of such a lattice $L$ is an $\alpha$-ideal if and only if $x \in J$ implies $(x]^{* *} \subseteq J$. For a prime ideal $P, O(P)=\{x$ $\in L: x \wedge y=0$ for some $y \in L \backslash P\}$ is an important $\alpha$-ideal associated with it. There are two ways of describing $O(P)$. Firstly, it is the intersection of all the minimal prime ideals contained within $P$. Secondly, it is the kernel of the congruence $\Psi(L \backslash P)$, where $\Psi(F)$ is the smallest congruence having filter $F$ as a congruence class. Both these descriptions are used in [2].

For an ideal $J, \Theta(J)$ denotes the congruence on $L$ which is defined as follows.

$$
x \equiv y(\Theta(J))(x, y \in L) \Leftrightarrow(x] \cap(j]^{*}=(y] \cap(j]^{*} \quad \text { for some } j \in J .
$$

We omit the easy verification of the fact that $\Theta(J)$ is a congruence; it is the natural generalization of the congruence $\Theta(J)\left(J\right.$ a kernel of a ${ }^{*}$-congruence in a distributive pseudocomplemented lattice) of [2, Theorem 1.5]. Of course, $J$ is contained within the kernel of $\Theta(J)$, and is the kernel of $\Theta(J)$ if and only if it is an $\alpha$-ideal. Even when $J$ is an $\alpha$-ideal, $\Theta(J)$ is, in general, strictly larger than $\Theta_{\text {Lat }}(J)$, the smallest lattice-congruences whose kernel is $J$. From [2, Theorem $1.5]$, the kernels of *-congruences of a distributive pseudocomplemented lattice are precisely its $\alpha$-ideals, and for such a kernel $J, \Theta(J)$ is the smallest*congruence having $J$ as its kernel.

Recall that a lattice $L$ is quasicomplemented if and only if it satisfies any of the following equivalent conditions: (1) the space of minimal prime ideals of $L$ is compact, (2) for each $x \in L$ there exists $y \in L$ such that $(x]^{* *}=(y]^{*}$, (3) for each $x \in L$, there is $y \in L$ such that $x \wedge y=0$ and $x \vee y$ is dense, i.e. $(x \vee y)^{*}=(0]$.

PROPOSITION 1.1. The following conditions on a lattice $L$ are equivalent:

(i) $L$ is quasicomplemented.

(ii) For each $\alpha$-ideal $J$, there is a filter $F$ such that $J=\operatorname{ker} \Psi(F)$.

(iii) For each $\alpha$-ideal $J, J=\operatorname{ker} \Psi(F(J))$, where $F(J)$ is the filter $F(J)$ $=\left\{x \in L:(z]^{*} \subseteq(x]^{* *}\right.$ for some $\left.z \in J\right\}$.

Proof. (i) $\Rightarrow$ (iii). $F(J)$ is a filter. For, if $\left(z_{1}\right]^{*} \subseteq\left(x_{1}\right]^{* *}$ and $\left(z_{2}\right]^{*} \subseteq\left(x_{2}\right]^{* *}$ then $\left(z_{1} \vee z_{2}\right]^{*}=\left(z_{1}\right]^{*} \cap\left(z_{2}\right]^{*} \subseteq\left(x_{1}\right]^{* *} \cap\left(x_{2}\right]^{* *}=\left(x_{1} \wedge x_{2}\right]^{* *}$ and if $x_{1}$ $\leqslant x$ also, then $\left(z_{1}\right]^{*} \subseteq\left(x_{1}\right]^{* *} \subseteq(x]^{* *}$. Let $w \in \operatorname{ker} \Psi(F(J)) ; w \wedge x=0$ for some $x \in F(J)$. Thus, $(z]^{*} \subseteq(x]^{* *}$ for some $z \in J$, and so $w \in(x]^{*} \subseteq(z]^{* *}$ $\subseteq J$, as $J$ is an $\alpha$-ideal. It remains to show that (i) implies $J \subseteq \operatorname{ker} \Psi(F(J))$. Let $x \in J$ and choose $y \in L$ such that $(x]^{* *}=(y]^{*}$. Then $x \wedge y=0$ and $(x]^{*} \subseteq(y]^{* *}$. Hence, $y \in F(J)$ and $x \in \operatorname{ker} \Psi(F(J))$.

(iii) $\Rightarrow$ (ii) is clear. Suppose (ii) holds, and let $x \in L$. Then $(x]^{* *}$ is an $\alpha$-ideal and so there is a filter $F$ such that $(x]^{* *}=\cup\left\{(f]^{*}: f \in F\right\}$. It immediately follows that $(x]^{* *}=(g]^{*}$ for some $g \in F$.

Proposition 1.2. The following conditions on a lattice L are equivalent:

(i) $L$ is pseudocomplemented.

(ii) For each $\alpha$-ideal $J, J=\operatorname{ker} \Psi\left(J_{*}\right)$, where $J_{*}$ is the filter $J_{*}=\left\{x \in L:(z]^{*}\right.$ $\subseteq(x]$ for some $z \in J\}$. 
(iii) For each $\alpha$-ideal $J, \Theta(J)=\Psi\left(J_{*}\right)$.

(iv) For each $\alpha$-ideal $J$, there is a filter $F$ such that $\Theta(J)=\Psi(F)$.

Proof. $J_{*}$ is always a filter, and (i) implies (iii) by [2, Theorem 1.5]. The implications (iii) $\Rightarrow$ (ii) and (iii) $\Rightarrow$ (iv) are obvious.

(iv) $\Rightarrow$ (iii). If $\Theta(J)=\Psi(F)$ then $F=\operatorname{coker} \Theta(J)$ and so $F=J_{*}$. Hence, $\Theta(J)=\Psi\left(J_{*}\right)$.

(ii) $\Rightarrow$ (i). By 1.1, (ii) implies that $L$ is quasicomplemented. Let $x \in L$ and choose $y \in L$ such that $(x]^{*}=(y]^{* *}$. As $x \wedge y \doteq 0, x \in(y]^{*}$ $=\operatorname{ker} \Psi\left(\left((y]^{*}\right)_{*}\right)$. Hence, $x \wedge z=0$ for some $z \in L$ such that $(a]^{*} \subseteq(z]$, where $a$ is a suitable member of $(y]^{*}$. Then, $(x]^{*}=(y]^{* *} \subseteq(a]^{*}$. Hence, $(x]^{*} \subseteq(z]$. It follows that $(x]^{*}=(z]$ and so $L$ is pseudocomplemented.

The proof is now complete.

Proposition 1.3. A lattice $L$ is pseudocomplemented if and only if, for each $\alpha$ ideal $J$ and each prime ideal $P, J_{*} \subseteq L \backslash P$ implies $J \subseteq O(P)$.

Proof. If $L$ is pseudocomplemented, $x \in J$, and $J_{*} \subseteq L \backslash P$, then $x^{*} \in J_{*}$ and so $x^{*} \in L \backslash P$. Hence, $x \in O(P)$, i.e. $J \subseteq O(P)$.

Now suppose the condition holds. Let $J$ be an $\alpha$-ideal. Of course, $\Psi\left(J_{*}\right)$ $\subseteq \Theta(J)$ always holds. Also, for any filter $F, \Psi(F)=\cap\{\Psi(G): F \subseteq G$, is a prime filter , by the dual of [2, Lemma 1.2 (ii)]. Also, in any lattice $L, \Theta(O(P)) \subseteq \Psi(L \backslash P)$, for any prime ideal $P$. Indeed, $x \equiv y(\Theta(O(P)))$ implies $(x] \cap(z]^{*}=(y] \cap(z]^{*}$ for some $z \in O(P)$, and so there is $w$ $\in(z]^{*} \cap(L \backslash P)$. Then, $x \wedge w=y \wedge w$ and $x \equiv y(\Psi(L \backslash P))$. Thus, $\Psi\left(J^{*}\right)$ $\supseteq \cap\left\{\Theta(O(P)): P\right.$ is a prime ideal, $\left.J_{*} \subseteq L \backslash P\right\}$. Hence, the condition implies that $\Psi\left(J^{*}\right) \supseteq \cap\{\Theta(O(P)): P$ is prime ideal, $J \subseteq O(P)\}$. (Note that $J$ $\subseteq O(P)$ always implies $J_{*} \subseteq L \backslash P$ and so the condition means $J \subseteq O(P)$ if and only if $J_{*} \subseteq L \backslash P$.) Hence, $\Psi\left(J_{*}\right) \supseteq \Theta(J)$. Thus $\Psi\left(J_{*}\right)=\Theta(J)$ and $L$ is pseudocomplemented by 1.2 .

Corollary 1.4. Let $L$ be pseudocomplemented, $J$ be an $\alpha$-ideal and $x \in L$. Then,

$$
\Theta(J)=\cap\{\Theta(O(P)): P \text { is a prime ideal, } J \subseteq O(P)\},
$$

and

$$
\Theta\left((x]^{*}\right)=\bigcap\left\{\Theta(O(P)): P \text { is a prime ideal, } x^{* *} \notin P\right\} .
$$

Proof. By 1.2 and 1.3, $\Theta(J)=\Psi\left(J_{*}\right)=\bigcap\{\Psi(L \backslash P): P$ is a prime ideal, $\left.J_{*} \subseteq L \backslash P\right\}=\bigcap\{\Psi(L \backslash P): P$ is a prime ideal, $J \subseteq O(P)\} \supseteq \cap\{\Theta(O(P)): P$ is a prime ideal, $J \subseteq O(P)\} \supseteq \Theta(J)$, for $\Psi(L \backslash P)$ always contains $\Theta(O(P))$ as was noted in the proof of 1.3.

Also, $\left((x]^{*}\right)_{*}=\left(x^{*}\right]_{*}=\left\{z \in L: x^{* *} \subseteq z\right\}$, as is easily seen. Hence, $\Theta\left((x]^{*}\right)$ $=\bigcap\left\{\Theta(O(P)): P\right.$ is a prime ideal, $\left.\left((x]^{*}\right)_{*} \subseteq L \backslash P\right\}=\bigcap\{\Theta(O(P)): P$ is a prime ideal, $\left.x^{* *} \in L \backslash P\right\}$.

The above propositions show how the relationship between $J, J_{*}, \Theta(J)$ and filters are almost characteristic of pseudocomplementation. Unfortunately, we do not know whether 1.4 is characteristic of pseudocomplementation. The following lemma is a consequence of a more general theorem [4, Theorem 2.4]. 
However, we give a simple and alternative self-contained proof.

LEMMA 1.5. Let $L$ be pseudocomplemented and $x_{1}, x_{2}, \ldots, x_{n}, a_{1}, a_{2}, \ldots, a_{n}$ $\in L$. Suppose $x_{i} \equiv x_{j}\left(\Theta\left(\left(a_{i} \wedge a_{j}\right]^{*}\right)\right)$ for all $i, j=1,2, \ldots, n$. Then there exists $x \in L$ such that $x \equiv x_{j}\left(\Theta\left(\left(a_{j}\right]^{*}\right)\right)$ for all $j=1,2, \ldots, n$.

Proof. Let $i, j \in\{1,2, \ldots, n\}$. Then, there exists $y \leqslant\left(a_{i} \wedge a_{j}\right)^{*}$ such that $x_{i} \wedge y^{*}=x_{j} \wedge y^{*}$. As $\left(a_{i} \wedge a_{j}\right)^{* *}=a_{i}^{* *} \wedge a_{j}^{* *} \leqslant y^{*}$, it follows that $x_{i}$ $\wedge a_{i}^{* *} \wedge a_{j}^{* *}=x_{j} \wedge a_{i}^{* *} \wedge a_{j}^{* *}$. Put $x=\left(x_{1} \wedge a_{1}^{* *}\right) \vee \cdots \vee\left(x_{n} \wedge a_{n}^{* *}\right)$. Then,

$$
\begin{aligned}
x \wedge a_{j}^{* *}= & \left(x_{1} \wedge a_{1}^{* *} \wedge a_{j}^{* *}\right) \vee \cdots \vee\left(x_{j} \wedge a_{j}^{* *}\right) \\
& \vee \cdots \vee\left(x_{n} \wedge a_{n}^{* *} \wedge a_{j}^{* *}\right) \\
= & \left(x_{j} \wedge a_{1}^{* *} \wedge a_{j}^{* *}\right) \vee \cdots \vee\left(x_{j} \wedge a_{j}^{* *}\right) \vee \cdots \vee\left(x_{j} \wedge a_{n}^{* *} \wedge a_{j}^{* *}\right) \\
= & x_{j} \wedge a_{j}^{* *} .
\end{aligned}
$$

Hence, $x \equiv x_{j}\left(\Theta\left(\left(a_{j}\right]^{*}\right)\right)$, for $j=1,2, \ldots, n$.

2. The sheaf representation. Let $S(L)$ be the set of all prime ideals of the pseudocomplemented lattice $L$. For $a \in L$, let $s(a)=\left\{P \in S(L): a^{* *} \notin P\right\}$. Notice that $s(a)=s\left(a^{* *}\right)$ and $s(a) \wedge s(b)=s(a \wedge b)$ for any $a, b \in L$. Also, $S(L)=\bigcup\{s(a): a \in L\}=s(1)$. Thus, $\{s(a): a \in L\}$ is a basis for a topology on $L$ which will be used throughout this section. We note that $\cup\left\{s\left(a_{i}\right): a_{i}\right.$ $\in L, i=1, \ldots, n, n \geqslant 1\} \subseteq s\left(a_{1} \vee a_{2} \vee \cdots \vee a_{n}\right)$. Also, if $S(L)$ $=\bigcup\left\{s\left(a_{k}\right): k \in K\right.$, an index set $\}$ then $V\left\{\left(a_{k}\right]^{* *}: k \in K\right\}=L$ and, hence $\left(a_{k_{1}}\right]^{* *} \vee \cdots \vee\left(a_{k_{m}}\right]^{* *}=L$ for some $k_{1}, k_{2}, \ldots, k_{m} \in K$. Hence, $s\left(a_{k_{1}}\right)$ $\vee \cdots \vee s\left(a_{k_{m}}\right)=L$. Thus, $S(L)$ is compact.

Let $\mathcal{P}(L)=\bigcup\{L / \Theta(O(P)): P \in S(L)\}$ and, for $a \in L$, let $\hat{a}$ be the Gelfand transform: $\hat{a}: S(L) \rightarrow \mathscr{P}(L), \hat{a}(P)=[a] \Theta(O(P))$, for each $P \in S(L)$.

Lemma 2.1. For any $a, b \in L,\{P \in S(L): \hat{a}(P)=\hat{b}(P)\}$ is open.

Proof. Let $Q \in\{P \in S(L): \hat{a}(P)=\hat{b}(P)\}$. Then, $a \equiv b(\Theta(O(Q)))$ and so $a \wedge y^{*}=b \wedge y^{*}$ for some $y \in O(Q)$. Then, $y^{*} \notin Q$, i.e. $Q \in s\left(y^{*}\right)$. If $Q_{1} \in s\left(y^{*}\right)$ then $y \in O\left(Q_{1}\right)$ and $a \equiv b\left(\Theta\left(O\left(Q_{1}\right)\right)\right)$. Hence, $Q \in s\left(y^{*}\right) \subseteq\{P$ $\in S(L): \hat{a}(P)=\hat{b}(P)\}$, and the lemma is proved.

Consider $L$ as an algebra $\left(L, \vee, \wedge,,^{*}, 0,1\right)$ of type $(2,2,1,0,0)$. Then, from general considerations [5, Lemma 2.1], if $\mathscr{P}(L)$ is endowed with the finest topology for which each $\hat{a}, a \in L$, is continuous, i.e. $\{\hat{a}(s(b)): a, b \in L\}$ is used as an open base, then $(\mathscr{P}(L), S(L))$ is a sheaf of distributive pseudocomplemented lattices considered as algebras of type $\langle 2,2,1,0,0\rangle$.

THEOREM 2.2. Let $L$ be pseudocomplemented. Then the map $a \rightarrow \hat{a}$ is an isomorphism of $L$ onto $\Gamma((\mathscr{P}(L), S(L)))$, the algebra of all global sections in the sheaf $(\mathscr{P}(L), S(L))$.

Proof. From Corollary 1.4, $\cap\{\Theta(O(P)): P \in S(L)\}=\omega$ and hence the map $a \rightarrow \hat{a}$ is a monomorphism. Let $\sigma \in \Gamma((\mathscr{P}(L), S(L)))$. Since two global sections agree at a point if and only if they agree on a basic open 
neighbourhood of the point, the compactness of $S(L)$ implies that there exist $x_{1}, x_{2}, \ldots, x_{n}, a_{1}, a_{2}, \ldots, a_{n} \in L$ such that $s(L)=s\left(a_{1}\right) \cup \cdots \cup s\left(a_{n}\right)$ and $\sigma(P)=\hat{x}_{i}(P)$, for each $P \in s\left(a_{i}\right)$ and $i=1,2, \ldots, n$. Thus, $x_{i}$ $\equiv x_{j}(\Theta(O(P)))$ for all $P \in s\left(a_{i}\right) \cap s\left(a_{j}\right)=s\left(a_{i} \wedge a_{j}\right)$ and $i, j=1,2, \ldots, n$. By 1.4, $x_{i} \equiv x_{j}\left(\Theta\left(\left(a_{i} \wedge a_{j}\right]^{*}\right)\right)$ for all $i, j=1,2, \ldots, n$. By 1.5, there exists $x \in L$ such that $x \equiv x_{j}\left(\Theta\left(\left(a_{j}\right]^{*}\right)\right)$ for each $j=1,2, \ldots, n$. Using 1.4 once more, it follows that $\hat{x}(P)=\hat{x}_{j}(P)$ for all $P \in s\left(a_{j}\right)$ and $j=1,2, \ldots, n$. Hence, $\sigma=\hat{x}$ and the theorem follows.

From [3, Theorem 3.4] and [2, Corollary 2.9] it follows that if $1 \leqslant n<\omega$ and $\mathscr{B}_{n}$ is the $n$th variety of distributive pseudocomplemented lattices then $L \in \mathscr{B}_{n}$ if and only if each of the stalks in the sheaf $(\mathscr{P}(L), S(L))$ is $m$-dense for some $1 \leqslant m \leqslant n-1$, where $L$ is $m$-dense if and only if, for any $x_{1}, x_{2}, \ldots, x_{m+1} \in L, x_{i} \wedge x_{j}=0$ for distinct $i, j=1,2, \ldots, m+1$, implies $x_{i}=0$ for some $i=1,2, \ldots, m+1$. Of course, $L$ is dense if and only if it is 1-dense.

We close by remarking that the topology that we imposed on $S(L)$ is analogous to the topology used by P. N. Stewart [6] in a different setting.

\section{REFERENCES}

1. W. H. Cornish, Annulets and $\alpha$-ideals in a distributive lattice, J. Austral. Math. Soc. 15 (1973), 70-77. MR 49 \# 8910.

2. - Congruences on distributive pseudocomplemented lattices, Bull. Austral. Math. Soc. 8 (1973), 161-179. MR 47 \#6573.

3. - n-normal lattices, Proc. Amer. Math. Soc. 45 (1974), 48-54. MR 49 \#4889.

4. - On the Chinese remainder theorem of H. Draskovicová, Mat. Casopis (submitted).

5. B. A. Davey, Sheaf spaces and sheaves of universal aigebras, Math. Z. 134 (1973), 275-290. MR 48 \#8345.

6. P. N. Stewart, A sheaf theoretic representation of rings with Boolean orthogonalities, Pacific J. Math. (to appear).

Department of Mathematics, Dalhousie University, Halifax, N.S., Canada

Current address: School of Mathematical Sciences, The Flinders University of South Australia, Bedford Park, 5042, South Australia, Australia 